\title{
Terapias medicamentosas propostas no manejo da COVID-19
}

\author{
Drug therapies proposed in the management of COVID-19
}

Terapias farmacológicas propouestas en el manejo de COVID-19

Larissa Pantoja Machado de Souza ${ }^{1 *}$, Wainnye Marques Ferreira ${ }^{1}$, Ketre Iranmarye Manos Nascimento ${ }^{1}$, Sheine Alves de Souza1, Vitória de Souza Lima1, Márcia Cristina Monteiro Guimarães'.

\begin{abstract}
RESUMO
Objetivo: Realizar uma revisão integrativa da literatura sobre o uso de medicamentos no manejo da COVID19 provocada pelo novo coronavirus. Métodos: Foi realizado uma revisão sistemática da literatura das publicações sobre COVID-19 e o tratamento associado nas bases de dados Scientific Eletronic Library Online (SciElo) e Publicação de Medicina (PubMed), utilizando critérios de inclusão para coleta de artigos e descritores "COVID" e "Medicamento". Resultados: Foram encontrados 6 artigos na SciElo, após critério de recorte temporal não foi excluído nenhum, contudo após critério de temática foi escolhido apenas um (1) artigo para análise. Já analisando o PubMed, foram encontrados 5.225 resultados e dentre esses, ficaram 2.200 após critério de recorte temporal, 1.173 após critério de texto completo sem custo para os autores desses, 76 continuaram no processo de escolha após passar pelo critério de assunto e temática e, por fim, foram encontrados 18 com maior nível de relevância dentro do PubMed. Considerações finais: Foram encontradas diversas pesquisas que tentam associar medicações com taxas de cura, contudo não foi possível encontrar uma pesquisa conclusiva que associasse um medicamento relacionado à cura do COVID-19.
\end{abstract}

Palavras-chave: Epidemiologia, COVID-19, Medicina.

\begin{abstract}
Objective: To carry out an integrative review of the literature on the use of drugs in the management of COVID19 caused by the new coronavirus. Methods: A systematic literature review of publications on COVID-19 and associated treatment in the Scientific Electronic Library Online (SciElo) and Publication of Medicine (PubMed) databases was performed, using inclusion criteria for the collection of articles and descriptors "COVID" and "Medicament". Results: Six articles were found in SciElo, after the criterion of time cut none was excluded, however, after thematic criterion, only one (1) article was chosen for analysis. Already analyzing PubMed, 5,225 results were found and among these, 2,200 were found after the time cut criterion, 1,173 after full text criterion at no cost to the authors of these, 76 continued in the selection process after going through the subject and thematic criterion and, finally, 18 were found with a higher level of relevance within PubMed. Final considerations: Several studies have been found that attempt to associate medications with cure rates, however it was not possible to find conclusive research that associated a drug related to the cure of COVID19.
\end{abstract}

Key words: Epidemiology, COVID-19, Medicine.

${ }^{1}$ Centro Universitário Metropolitano da Amazônia (UNIFAMAZ), Belém - PA.

*E-mail: larissapantoja@yahoo.com.br

SUBMETIDO EM: 2/2021

ACEITO EM: 3/2021

PUBLICADO EM: 4/2021 


\section{RESUMEN}

Objetivo: Realizar una revisión integradora de la literatura sobre el uso de fármacos en el manejo del COVID19 causado por el nuevo coronavirus. Métodos: Se realizó una revisión bibliográfica sistemática de publicaciones sobre COVID-19 y tratamiento asociado en las bases de datos Scientific Electronic Library Online (SciElo) y Publication of Medicine (PubMed), utilizando criterios de inclusión para la colección de artículos y descriptores "COVID" y "Medicamento". Resultados: Se encontraron seis artículos en SciElo, luego del criterio de corte de tiempo no se excluyó ninguno, sin embargo, luego del criterio temático, solo se eligió un (1) artículo para el análisis. Ya analizando PubMed se encontraron 5.225 resultados y de estos, 2.200 se encontraron después del criterio de corte de tiempo, 1.173 después del criterio de texto completo sin costo para los autores de estos, 76 continuaron en el proceso de selección luego de pasar por el tema y los criterios temáticos, y finalmente, 18 se encontraron con mayor nivel de relevancia dentro de PubMed. Consideraciones finales: Se encontraron varios estudios que intentan asociar medicamentos con tasas de curación, sin embargo, no fue posible encontrar investigaciones concluyentes que asociaran un medicamento relacionado con la cura de COVID-19.

Palabras clave: Epidemiología, COVID-19, Medicina.

\section{INTRODUÇÃO}

O Síndrome da Angústia Respiratória causada pelo Coronavírus (SARS-CoV-2) é um novo tipo de coronavírus pertencente a uma grande família de vírus que são comuns em muitas espécies de animais, entre ele estão o gato, gado e morcego. No entanto, poucas são as evidências da transmissão dos coronavírus entre animais e humanos. A COVID-19, doença causada pelo SARS-CoV-2, foi descoberta no final de 2019, em Wuhan na China, que com sua disseminação e alto poder de transmissão de pessoa a pessoa, tornouse, em poucos meses, um problema mundial de saúde pública (BRASIL, 2020).

Esse novo coronavírus apresenta uma variação no quadro clínico em que pode apresentar infecções assintomática a quadros graves de infecção. Segundo a Organização Mundial da Saúde (OMS), (80\% das pessoas infectadas com COVID-19 podem apresentar poucos ou nenhum sintoma, os outros $20 \%$ dos infectados necessitam de atendimento hospitalar por apresentarem dificuldade respiratória, dos quais aproximadamente $5 \%$ podem necessitar de suporte ventilatório (BRASIL, 2020).

De acordo com um estudo realizado no Reino Unido, dos 17.278 .392 adultos estudados, 10.926 morreram por complicações da COVID-19, apontando, nessa pesquisa, alguns fatores de risco do novo coronavírus. Verificou-se, segundo os pesquisadores, que o sexo masculino tem maior risco de morte pelo vírus, da mesma forma que idosos, diabéticos, pessoas com asma severa, assim como a obesidade, doenças respiratórias, cardiopatia crônica, Acidente Vascular Cerebral (AVCs), demência e outros problemas neurológicos. De fato, até o mês de maio, os estudos não mostraram evidências sobre a maior mortalidade nos homens do que nas mulheres e apenas surgiu a hipótese de que seria uma combinação de três fatores como hábitos, higiene e hormônios (BRASIL, 2020; OPAS, 2020).

Apesar dos fatores de risco favorecerem o contágio e o agravamento da doença, qualquer pessoa pode ser infectada com o novo coronavírus, porém a apresentação dos sintomas varia de pessoa para pessoa. Os sintomas mais comuns da COVID-19 são hipertermia, cansaço e tosse seca, porém algumas pessoas apresentam congestão nasal, cefaleia, dores, conjuntivite, dor de garganta, diarreia, anosmia, ageusia, erupção cutânea na pele ou descoloração dos dedos das mãos e dos pés. Tais sintomas apresentam-se, geralmente, de forma leve e gradual. A maioria das pessoas infectadas apresentam sintomas leves e/ou moderados, dessa forma não precisam de tratamento hospitalar, no entanto uma em cada seis pessoas infectadas apresenta o agravamento do quadro clínico e desenvolve dificuldade respiratória, geralmente as pessoas pertencentes aos grupos de risco citados acima (OPAS, 2020).

Com base em estudos recentes, a transmissão do novo coronavírus ocorre por contato de pessoa a pessoa e pelo ar com secreções contaminadas por meio de gotículas de saliva, espirro, tosse, toque ou aperto de 
mão, contato com objetos ou superfícies contaminadas e, logo em seguida, o contato com a boca, nariz ou olhos (BRASIL, 2020).

O período em que os sintomas levam para aparecer é em média de 5 a 12 dias, enquanto que sua transmissibilidade é em média 7 dias após o início dos sintomas, contudo, estudos indicam que a transmissão do novo coronavírus possa ocorrer mesmo sem o aparecimento dos sintomas. Além disso, é importante ressaltar que não há informações satisfatórias de quantos dias anteriores ao início dos sinais e sintomas uma pessoa infectada passa a transmitir o vírus (BRASIL, 2020).

Segundo as orientações da OMS, é necessário seguir alguns cuidados para prevenir o contágio da COVID19 como: lavar as mãos com água e sabão ou higienizá-las com álcool a 70\%; manter o distanciamento de pelo menos 1 metro das outras pessoas, quer elas estejam com alguns sintomas ou não; evitar tocar com as mãos no nariz, boca ou olhos, visto que as mãos estão em constante contato com superfícies contaminadas; ao tossir ou espirrar cobrir a boca ou nariz com a parte interna do cotovelo ou com lenço de papel (SOCIEDADE BRASILEIRA DE PNEUMOLOGIA E TISIOLOGIA, 2020).

Muito tem sido proposto em relação às terapias medicamentosas da COVID-19, mas não há, até o momento, comprovação científica de que esses medicamentos sejam eficazes e seguros para o tratamento da doença. Há diversas terapias sendo utilizadas para o tratamento, mas necessitando de uma avaliação apropriada de segurança e efetividade e que vale ressaltar que a grande maioria das terapias não possui registro em bula para uso em COVID-19. Muitas informações utilizadas nesses tratamentos são baseadas em evidências de infecções anteriores por Coronavírus, mas são informações limitadas, pois o vírus teve grandes mutações (MANISHA P, 2020).

Além disso, a potencial proporção que o vírus alcançou dificulta ainda mais e limita as opções terapêuticas possíveis para combater a doença e, devido ao crescente número de infectados e mortes pela doença, o desenvolvimento de terapêuticas eficazes e seguras tornou-se uma preocupação global preferencial para o controle e a cura da infecção por COVID-19 (DANTE J, 2020).

O objetivo do presente artigo foi realizar uma revisão sistemática da literatura sobre 0 uso de medicamentos no manejo do novo coronavírus (COVID-19).

\section{MÉTODOS}

Foi realizado uma revisão integrativa da literatura das publicações sobre o novo coronavírus (COVID-19) e o tratamento associado nas bases de dados Scientific Eletronic Library Online (SciElo) e Publicações de Medicina (PubMed) utilizando critérios de inclusão para coleta de artigos.

Foram coletados artigos que atendessem ao critério de inclusão que estivessem dentro do recorte temporal (publicados em 2020), disponibilidade de texto completo sem custo para os autores e ao critério de assunto ou temática (terapêutica em covid) em algum momento do texto utilizando o operador booleano "AND" nos descritores do Descritores em Ciências da Saúde (DECS) "COVID" e "Medicamento" para SciElo e os descritores MeSH "Coronavirus" e "Therapeutic" para busca em PubMed acolhendo artigos que estivessem nas línguas portuguesa, inglesa ou espanhola.

Os critérios de exclusão foram artigos publicados fora do recorte temporal proposto, indisponibilidade do texto completo ou que tivesse algum custo para os autores, que não abordassem ao assunto ou temática em algum momento do texto e que fossem publicados em outras bases de dados que não as escolhidas (Figura 1). 
Figura 1 - Fluxograma de metodologia.

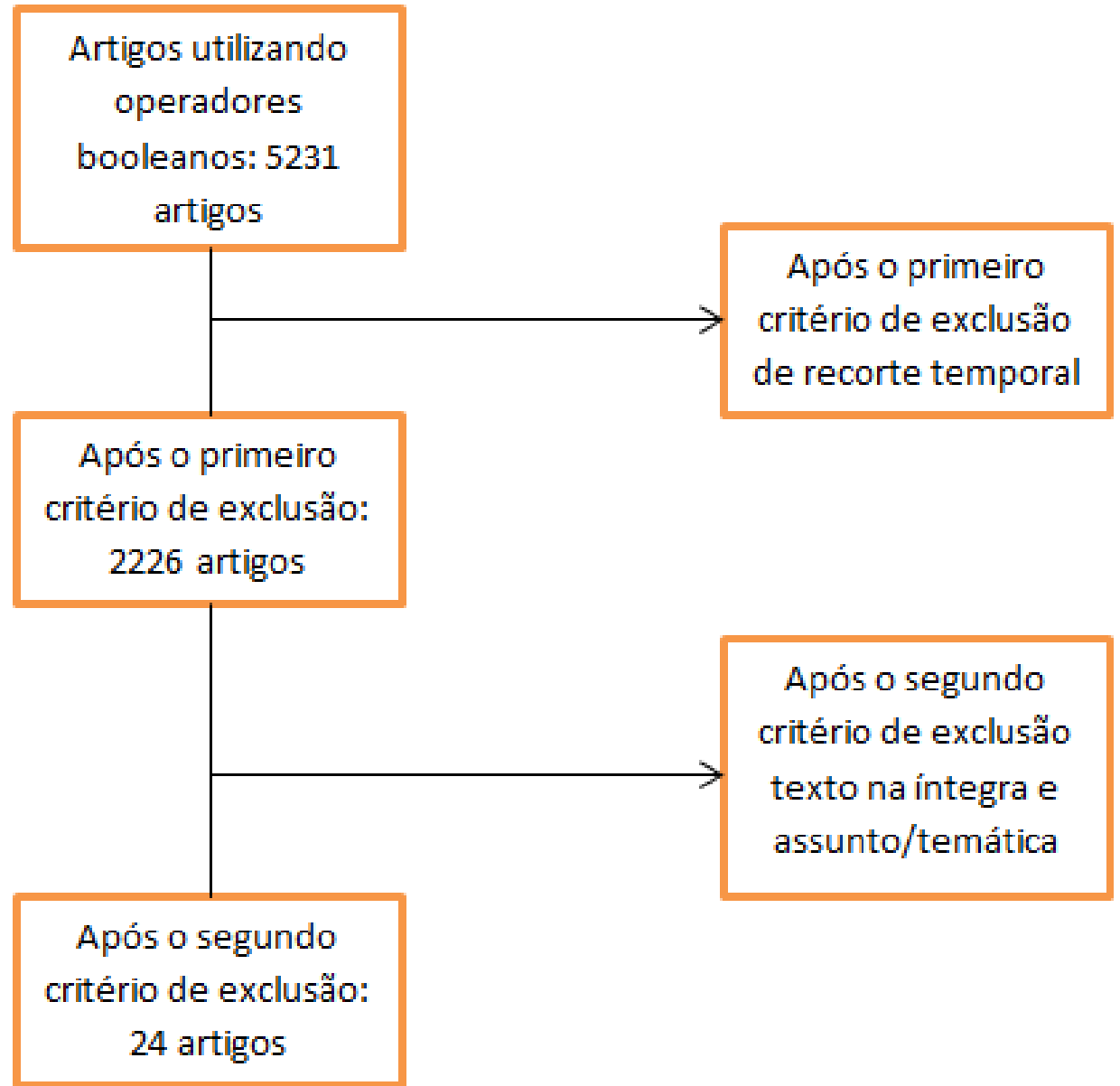

Fonte: Souza LPM, et al., 2021.

\section{RESULTADOS}

Após coleta de dados por meio dos critérios de inclusão, foram encontrados 6 artigos na SciElo, após critério de recorte temporal não foi excluído nenhum, contudo após critério de temática foi escolhido apenas um (1) artigo para análise.

Já analisando o PubMed, foram encontrados 5.225 resultados e dentre esses, ficaram 2.200 após critério de recorte temporal, 1.173 após critério de texto completo sem custo para os autores desses, 76 continuaram no processo de escolha após passar pelo critério de assunto e temática e, por fim, foram encontrados 18 com maior nível de relevância dentro do PubMed (Quadro 1). 
Quadro 1 - Quadro com características dos artigos escolhidos para a revisão sistemática da literatura.

\begin{tabular}{|c|c|c|c|c|}
\hline Procedência & Título do artigo & Autores & Periódico & Considerações/ Temática \\
\hline Pubmed & $\begin{array}{l}\text { Efficacy of integrative Traditional Chinese } \\
\text { and Western medicine for the treatment of } \\
\text { patients infected with } 2019 \text { novel } \\
\text { coronavirus (COVID-19) }\end{array}$ & Dan L, et al., 2020 & Medicine, 2020; 99:29 & $\begin{array}{l}\text { Eficácia do tratamento integrativo tradicional } \\
\text { chinês e ocidental em pacientes com } \\
\text { COVID-19 }\end{array}$ \\
\hline Pubmed & $\begin{array}{l}\text { Medications in COVID-19 patients: } \\
\text { summarizing the current literature from an } \\
\text { orthopaedic perspective }\end{array}$ & Si HST, et al., 2020 & $\begin{array}{l}\text { Ortopedia Internacional } \\
\text { (SICOT), 2020; 44: 1599- } \\
1603\end{array}$ & $\begin{array}{l}\text { Medicamentos comuns prescritos em } \\
\text { cirurgia ortopédica e suas potenciais } \\
\text { implicações em pacientes COVID-19. }\end{array}$ \\
\hline Pubmed & $\begin{array}{l}\text { Efficacy and safety of convalescent plasma } \\
\text { for severe COVID-19 based on evidence in } \\
\text { other severe respiratory viral infections: a } \\
\text { systematic review and meta-analysis }\end{array}$ & $\begin{array}{l}\text { Niveditha DA, et al., } \\
2020\end{array}$ & $\begin{array}{lr}\text { Canadian } & \text { Medical } \\
\text { Association } & \text { Journal } \\
(\text { CMAJ) }, 2020 ; 6 ; 192: 745- \\
55\end{array}$ & $\begin{array}{l}\text { Diretrizes baseadas em evidências para o } \\
\text { tratamento de pacientes com COVID-19 não } \\
\text { grave e grave que fornecem estratégias para } \\
\text { evitar o uso excessivo de terapias altamente } \\
\text { elogiadas, mas não estabelecidas. }\end{array}$ \\
\hline Pubmed & $\begin{array}{l}\text { Drug targets for corona virus: A systematic } \\
\text { review }\end{array}$ & $\begin{array}{l}\text { Manisha PP, et al., } \\
2020\end{array}$ & $\begin{array}{l}\text { Indian J Pharmacol. 2020; } \\
\text { 52(1): 56-65 }\end{array}$ & $\begin{array}{l}\text { Uso de medicamentos off-label, antivirais, } \\
\text { anticorpos, vitaminas e farmacoterapia } \\
\text { chinesa }\end{array}$ \\
\hline Pubmed & $\begin{array}{l}\text { Review of trials currently testing treatment } \\
\text { and prevention of COVID-19 }\end{array}$ & $\begin{array}{l}\text { Fragkou PC, et al., } \\
2020\end{array}$ & $\begin{array}{l}\text { Clinical and Microbiological } \\
\text { and Infection, 2020, 26(8): } \\
9\end{array}$ & $\begin{array}{l}\text { Determina análise da doença COVID }-19 \\
\text { com embasamento de pesquisa em banco } \\
\text { de dados de literatura. }\end{array}$ \\
\hline Pubmed & $\begin{array}{l}\text { A systematic review and meta-analysis of } \\
\text { the efficacy and safety of arbidol in the } \\
\text { treatment of coronavirus disease } 2019\end{array}$ & Xuemei W, et al., 2020 & $\begin{array}{l}\text { Medicine, 2020; 99(30): } \\
\text { e21402. }\end{array}$ & $\begin{array}{l}\text { Expõe sobre toda estrutura composição e } \\
\text { mecanismo de ação do COVID-19 e } \\
\text { pesquisas que abordam um possível } \\
\text { tratamento eficaz. }\end{array}$ \\
\hline Pubmed & $\begin{array}{l}\text { Perspectives on monoclonal antibody } \\
\text { therapy as potential therapeutic } \\
\text { intervention for Coronavirus disease-19 } \\
\text { (COVID-19) }\end{array}$ & $\begin{array}{l}\text { Balamurug S, et al., } \\
2020\end{array}$ & $\begin{array}{l}\text { Medicine, 2020; 38(1):10- } \\
18 .\end{array}$ & $\begin{array}{l}\text { Mostra resumos, na revisão sistemática, os } \\
\text { resultados clínicos do uso de drogas } \\
\text { antirretrovirais para prevenção e tratamento } \\
\text { COVID-19 e os ensaios clínicos planejados. }\end{array}$ \\
\hline
\end{tabular}




\begin{tabular}{|c|c|c|c|c|}
\hline Procedência & Título do artigo & Autores & Periódico & Considerações/ Temática \\
\hline Pubmed & $\begin{array}{l}\text { Systematic review of the efficacy and } \\
\text { safety of antiretroviral drugs against SARS, } \\
\text { MERS or COVID-19: initial assessment }\end{array}$ & Nathan F, et al., 2020 & $\begin{array}{l}J \text { Int AIDS Soc, 2020; } \\
\text { 23(4):e25489. }\end{array}$ & $\begin{array}{l}\text { Avalia a segurança e a eficácia do HCQ } \\
\text { sozinho ou em combinação em termos de } \\
\text { "tempo de cura clínica", "cura virológica", } \\
\text { "morte ou agravamento clínico da doença", } \\
\text { "progressão radiológica" e segurança }\end{array}$ \\
\hline Pubmed & $\begin{array}{l}\text { Virological and clinical cure in COVID-19 } \\
\text { patients treated with hydroxychloroquine: A } \\
\text { systematic review and meta-analysis }\end{array}$ & Sarma P, et al., 2020 & $\begin{array}{l}\text { Journal of Medical Virology, } \\
\text { 2020; 92(7): 776-785 }\end{array}$ & $\begin{array}{l}\text { Compara os efeitos dos tratamentos para } \\
\text { COVID-19 }\end{array}$ \\
\hline Pubmed & $\begin{array}{l}\text { Drug treatments comparision of plascm } \\
\text { convalescent for } \\
\text { covid- } 19\end{array}$ & $\begin{array}{l}\text { Siemieniuk RJ, et al., } \\
2020\end{array}$ & $\begin{array}{l}\text { British Medical Journal } \\
\text { (BMJ), 2020; e2980 }\end{array}$ & $\begin{array}{l}\text { Explora a eficácia do tratamento e prever os } \\
\text { efeitos potenciais do plasma convalescente } \\
\text { com COVID-19 }\end{array}$ \\
\hline Pubmed & $\begin{array}{l}\text { Drug treatments for covid-19: living } \\
\text { systematic review and network meta- } \\
\text { analysis }\end{array}$ & Sun YM, et al., 2020 & $\begin{array}{l}\text { International Journal of } \\
\text { Infectious Diseases, 2020; } \\
\text { 98: 334-346 }\end{array}$ & $\begin{array}{l}\text { Os efeitos anti-inflamatórios da } \\
\text { imunossupressão poderiam diminuir a } \\
\text { expressão clínica da doença. Tacrolimus e } \\
\text { ciclosporina, a maioria drogas comumente } \\
\text { usadas para manutenção da } \\
\text { imunossupressão após o transplante de } \\
\text { órgãos sólidos, reduzem a produção de } \\
\text { interleucina-2. }\end{array}$ \\
\hline Pubmed & $\begin{array}{l}\text { Immunosuppression drug-related and } \\
\text { clinical manifestation of Coronavirus } \\
\text { disease 2019: A therapeutical hypothesis }\end{array}$ & $\begin{array}{l}\text { Romanelli A, et al., } \\
2020\end{array}$ & $\begin{array}{l}\text { Am J Transplant. 2020; 00: } \\
1-2\end{array}$ & $\begin{array}{l}\text { A imunoterapia é um intervenção de opção } \\
\text { terapêutica eficiente contra COVID-19 e o } \\
\text { principais métodos a este respeito, como o } \\
\text { uso de imunoglobulinas e plasma terapia } \\
\text { melhorou os resultados clínicos em } \\
\text { pacientes infectados com COVID-19 }\end{array}$ \\
\hline Pubmed & $\begin{array}{l}\text { The possible of immunotherapy for COVID- } \\
\text { 19: A systematic review }\end{array}$ & Akram AS, et al., 2020 & $\begin{array}{l}\text { International } \\
\text { Immunopharma, 2020; 83: } \\
\text { e106455 }\end{array}$ & $\begin{array}{l}\text { Corticosteróides podem reduzir a } \\
\text { mortalidade para pacientes com COVID-19 e } \\
\text { ARDS. }\end{array}$ \\
\hline
\end{tabular}




\begin{tabular}{|c|c|c|c|c|}
\hline Procedência & Título do artigo & Autores & Periódico & Considerações/ Temática \\
\hline Pubmed & $\begin{array}{l}\text { Efficacy and safety of corticosteroids in } \\
\text { COVID-19 based on evidence for COVID- } \\
\text { 19, other coronavirus infections, influenza, } \\
\text { community-acquired pneumonia and acute } \\
\text { respiratory distress syndrome: a } \\
\text { systematic review and meta-analysis }\end{array}$ & Zhikang Y, et al., 2020 & 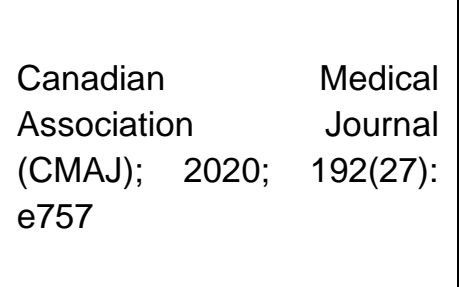 & $\begin{array}{l}\text { O perfil de efeitos colaterais de remdesivir } \\
\text { permanece igualmente não bem definido. } \\
\text { Até alta qualidade estudos relatam melhorias } \\
\text { significativas com a administração de } \\
\text { remdesivir IV }\end{array}$ \\
\hline Pubmed & $\begin{array}{l}\text { Remdesivir for the Treatment of COVID-19: } \\
\text { A Systematic Review of the Literature }\end{array}$ & Musa A, et al., 2020 & $\begin{array}{lr}\text { Western } & \text { Journal of } \\
\text { Emergency } & \text { Medicine; } \\
2020 ; 20(4) & \end{array}$ & $\begin{array}{l}\text { Essa revisão aborda sobre a compreensão } \\
\text { da imunopatogênese do SARS-CoV2 e } \\
\text { abordando os benefícios, desafios e } \\
\text { considerações sobre os NAbs. }\end{array}$ \\
\hline Pubmed & $\begin{array}{l}\text { Tocilizumab for severe COVID-19: a } \\
\text { systematic review and meta-analysis }\end{array}$ & $\begin{array}{l}\text { Shao-Huan L, et al., } \\
2020\end{array}$ & $\begin{array}{l}\text { Medicine, } 2020 ; \quad 56(3): \\
\text { e106103 }\end{array}$ & $\begin{array}{l}\text { A revisão avalia a eficácia de tocilizumabe } \\
\text { para o tratamento da COVID-19. }\end{array}$ \\
\hline Pubmed & $\begin{array}{l}\text { Treatment for severe acute respiratory } \\
\text { distress syndrome from COVID- } 19\end{array}$ & $\begin{array}{l}\text { Mathhay MAJ, et al., } \\
2020\end{array}$ & $\begin{array}{l}\text { Lancet Respir Med, 2020; } \\
8(5): 433-434\end{array}$ & $\begin{array}{l}\text { Expõe os desafios da implementação da } \\
\text { oxigenação por membrana extracorpórea } \\
\text { (ECMO) em pacientes com COVID-19. }\end{array}$ \\
\hline Scielo & $\begin{array}{l}\text { Agentes potencialmente terapêuticos } \\
\text { contra el SARS-CoV-2: Revisión de la } \\
\text { evidencia }\end{array}$ & $\begin{array}{l}\text { Bendezu-Quispe G, et } \\
\text { al., } 2020\end{array}$ & $\begin{array}{l}\text { Rev Peru Med Exp Salud } \\
\text { Publica. } 2020 ; 37(2): 320-6\end{array}$ & $\begin{array}{l}\text { Analisa evidencias sobre agentes } \\
\text { potencialmente terapêuticas contra a SARS } \\
\text { CoV-2. }\end{array}$ \\
\hline
\end{tabular}

Fonte: SOUZA LPN, et al., 2021. 


\section{DISCUSSÃO}

Atualmente não existe um antiviral específico para o tratamento de patologias associadas ao Sars-CoV. A maioria das estratégias de tratamento concentra-se no manejo sintomático e na terapia de suporte apenas. Alguns agentes terapêuticos que estão em desenvolvimento ou sendo usados off-label são a ribavirina, o interferon (IFN) $-\alpha$ e o ácido micofenólico. Existem muitos artigos de jornal citando a eficácia dos medicamentos anti-HIV: ritonavir, lopinavir, sozinho ou em combinação com oseltamivir, remdesivir e cloroquina; e entre estes, ritonavir, remdesivir e cloroquina mostraram eficácia a nível celula que ainda precisam de suporte experimental e validação (MANISHA P, 2020).

Já em relação à amiodarona observou-se que fica acumulada nas organelas ácidas. Vacúolos na exposição à amiodarona mostram alterações nas organelas intracelulares, especialmente o aumento dos endossomos tardios. Em ambiente in vitro, a amiodarona inibiu a infecção por coronavírus em células Vero (MANISHA P, 2020).

Outros agentes farmacoterapêuticos importantes que estão sendo avaliados são vitamina $C$ em altas doses, favipiravir, adalimumabe, diidro-artemisinina piperaquina, leflunomida, dipiridamol, suramina sódica, IFN-alfa 2b, partículas de Huo-Shen, injeção de Xiyanping, injeção de Shen-Fu, etc., muitos dos quais são oriundos da medicina tradicional chinesa. $O$ uso de células-tronco também é avaliado com frequência (MANISHA P, 2020).

Das opções de tratamento propostas para COVID-19, o plasma convalescente tem evidências que sugerem um benefício de mortalidade para infecção pelo vírus Ebola. Esta intervenção também foi testada em outras infecções respiratórias virais agudas graves. O "plasma convalescent" refere-se ao plasma obtido de indivíduos recentemente recuperado de uma doença viral, que se espera conter os níveis mais altos de anticorpos policlonais dirigidos contra o vírus. Da mesma forma, o "plasma hiperimune" é coletado de doadores exibindo altos títulos de anticorpos neutralizantes, independente do tempo decorrido desde a doença viral. Os autores usaram os termos indistintamente, e porque a neutralização viral é apenas um dos mecanismos postulados pelos quais os anticorpos exercem seu efeito antiviral, a importância da distinção entre os 2 produtos permanece obscura (DANTE J, 2020).

Os estudos existentes fornecem apenas evidências de qualidade muito baixa para apoiar as inferências sobre os benefícios do plasma convalescente em pacientes com COVID-19. Com base em evidências de baixa qualidade, não há nenhuma sugestão de que o plasma convalescente causaria qualquer evento adverso em pacientes com COVID-19 (SHARON TSH, 2020).

O mérito do plasma convalescente é a sua aparente baixa taxa de graves efeitos adversos. Embora isso possa ser uma vantagem sobre outras terapias não comprovadas para COVID-19, é insuficiente para justificar seu uso sem evidência associada de eficácia. Um banco de plasma convalescente consumiria recursos que, enquanto se aguarda evidência confiável de efeitos positivos, poderiam ser melhor alocados em outro lugar. $O$ fato de os médicos terem começado a usar plasma convalescente em pacientes com COVID-19 em hospitais fora dos ensaios clínicos torna urgente abordar seu valor terapêutico. O uso de plasma convalescente deve, portanto, aguardar evidências de alta qualidade de ensaios randomizados, idealmente testando o efeito do plasma (DANTE J, 2020).

Outra pesquisa analisa medicamentos que incluem analgesia, anti-inflamatórios, esteroides, anticoagulantes, antibióticos, vitamina B, vitamina $C$ e vitamina $D$. Paracetamol foi considerado a analgesia de primeira linha de acordo com a escala de dor da Organização Mundial da Saúde (OMS). Continua recomendado como a primeira linha de analgesia e antipirético para pacientes COVID-19. Nenhum estudo publicado relataram um efeito do paracetamol em pacientes com COVID-19. A maior parte da discussão sobre analgesia, anti-inflamatórios e COVID-19 gira em torno dos AINEs (SHARON TSH, 2020).

No entanto, as investigações subsequentes revelaram que não há estudos publicados que tenham encontrado uma associação real entre o uso de medicamentos anti-inflamatórios e uma maior incidência ou agravamento das infecções por COVID-19. Na verdade, um estudo anterior in vitro e in vivo que investigou 0 
efeito da indometacina no coronavírus sugeriu que a indometacina exibiu potente atividade antiviral por inibição da replicação viral e protegeu a célula hospedeira de danos induzidos por vírus. Relatórios e diretrizes subsequentes, incluindo a declaração da OMS, publicaram, portanto, declarações oficiais recomendando não evitar o uso de AINEs com base na evidência disponível atual (SHARON TSH, 2020).

Já sobre os antibióticos, os únicos que são discutidos em relação a COVID-19 são macrolídeos e tetraciclinas pois foram investigados e discutidos em várias publicações em vista de seu potencial efeito terapêutico em COVID-19. No entanto três estudos observacionais foram publicados sobre o uso de hidroxicloroquina e azitromicina em pacientes COVID-19 onde foram observadas a administração de hidroxicloroquina sozinha e hidroxicoroquina associada à azitromicina. Os resultados, no entanto, foram inconclusivos pois dois estudos relataram resultados positivos com o uso de hidroxicloroquina, especialmente quando complementado com azitromicina, enquanto um estudo não demonstrou qualquer atividade antiviral com os medicamentos. A Tetraciclina foi apontada como um potencial agente terapêutico para COVID-19 em vista de sua atividade contra metaloproteinases de matriz e citocinas inflamatórias, que são cruciais para a atividade viral, mas seus efeitos não foram encontrados em qualquer estudo publicado até agora (SHARON TSH, 2020).

A Medicina tradicional chinesa (MTC) integrada com a medicina ocidental (convencional) foi proposta como uma opção terapêutica para COVID-19 na China. Em 23 de março de 2020, um total de 74.187 casos confirmados na China ( $91,5 \%$ do total confirmado casos) foram tratados com MTC e a taxa efetiva total atingiu mais de $90 \%$. Os dados disponíveis indicam que a integração MTC com a medicina ocidental pode aliviar os sintomas clínicos, diminuir a duração da febre, facilitar a melhora radiológica, evitar que casos leves se transformem em casos graves, encurte o tempo de internação hospitalar e reduzir a taxa de mortalidade (MANISHA P, 2020).

Remédios chineses patenteados e fórmulas à base de ervas, como Jinhua, Grânulo Qinggan, Cápsula Lianhua Qingwen, Injeção Xuebijing, Qingfei Paidu Tang, Huashi Baidu Fang, Xuanfei Baidu Fang, Maxing Ganshi Tang, Yinqiao San, Mahuang Shengma Tang, Xiao Chaihu Tang, Da Yuan Yin, Gancao Ganjiang Tang e Shegan Mahuang Tang foram prescritos com base em diferenciação de padrões. A evidência clínica existente indica que medicina integrativa chinesa e ocidental pode ser uma abordagem terapêutica potente para COVID-19 (MANISHA P, 2020).

Dentre vários estudos explorando medicamentos e seus componentes que já atuavam ao combate de outras doenças, foram submetidos à análise para ação com o coronavírus 2 (SARS-CoV-2), verificando se alcançavam o objetivo de fortalecimento a resposta imune do hospedeiro contra o vírus, inibição da replicação e controle da liberação dos mediadores inflamatórios, sendo exposta sua eficácia e desuso. Nesse sentido, esses a título de informação: lopinavir (inibidor da protease ativo contra a infeção pelo vírus HIV1), remdesivir (um análogo de adenosina com propriedades antivirais de amplo espectro), favipiravir (um análogo de nucleosídeo que inibe RNA polimerase, inicialmente aprovado para tratamento do vírus influenza), azvudina (um análogo da azidocitidina que inibe a transcriptase reversa viral, eficaz contra HIV e os vírus da hepatite $\mathrm{B}$ e C), a cloroquina e a hidroxicloroquina ( licenciadas no tratamento da malária e doenças autoimunes ) associação com azitromicina, imunomoduladores e medicamentos anti-inflamatórios e terapias celulares (FRAGCOU P, 2020).

Devido ao grande impacto mundial causado pelo vírus, é relevante o desenvolvimento de imunoterapia passiva em anticorpos monoclonais para fornecer uma resposta rápida de ação a este vírus, desse modo impulsionando a comunidade de pesquisa fazer um progresso (XUEMEI WI, 2020; SHANMUGARAJ B, 2020).

Na pandemia de Covid-19 diversos medicamentos têm sido utilizados como tratamento, um deles são os antirretrovirais, que atuam como inibidores da protease, pois inibem as enzimas que ativam as glicoproteínas do envelope como parte do processo de entrada do vírus nas células. O uso de lopinavir/ ritonavir que já havia utilizado em testes in vitro e outros em pacientes com SARS, foram analisados para sua eficiência com SARS, MERS, COVID-19. Obtendo como resultado de identificação da revisão sistemática dos ensaios clínicos randomizados sem benefícios clínicos e dos 21 estudos observacionais inconclusivos com resultado muito baixo para a Covid-19 (NATHAN F, 2020). 
Outra forma de tratamento da COVID-19 é relatada no estudo de Sarma TSH et al (2020), no qual aborda efeitos da terapia com hidroxicloroquina comparados a outros tipos de tratamento. A terapia com HCQ pode ter efeito benéfico ao apresentar redução no número de casos de progressão radiológica, com perfil de eventos adversos comparável aos demais tratamentos envolvidos no estudo. Além disso, em relação a termos de tempo para o controle da temperatura corporal e vários dias de tosse, há evidências de possíveis benefícios. Por outro lado, não houve nenhuma observação relacionada à cura virológica no dia seis e sete após o início do tratamento e morte combinada ou agravamento do quadro clínico. Outro achado no estudo envolve a combinação de $\mathrm{HCQ}$ + Azi, nesse caso, até o momento, não há evidências que comprove, de fato, o seu benefício. Dessa maneira, é evidente que há uma grande necessidade de mais estudos clínicos para, dessa forma, chegar a uma conclusão definitiva (PHULEN S, 2020).

A certeza das evidências é muito baixa para a maioria das comparações entre os tratamentos medicamentosos da COVID-19 até o momento. Observou-se que a ação dos glicocorticoides resulte em uma possível redução dos riscos de morte e ventilação mecânica, assim como a redução do tempo de internação hospitalar. Com relação a remdesivir, não há evidências consistentes, apenas certeza moderada quanto a redução do tempo de resolução dos sintomas e a duração da ventilação mecânica, porém permanece incerto seu impacto sobre outros desfechos para o paciente, como, por exemplo, a mortalidade (REED S, 2020).

Atualmente, evidência relacionada à $\mathrm{HCQ}$ sugere a possibilidade de não reduzir o risco de morte, ventilação mecânica ou permanência da hospitalização. Apesar da redução do tempo para a resolução dos sintomas aos pacientes que receberam $\mathrm{HCQ}$, comparados aos que receberam tratamento padrão, é improvável que esse tipo de terapia apresente outros benefícios. Diante desse contexto, fica evidente que os efeitos da maioria dos tratamentos da COVID-19 são incertos, e que não há evidências consistentes sobre quaisquer outras intervenções que resultem em benefícios ou prejuízos à resolução da doença (REED $S$, 2020).

Um artigo aborda sobre uma terapia alternativa para pacientes com insuficiência respiratória aguda, que é a oxigenação por membrana extracorpórea (ECMO), qual demonstra excelentes resultados. Contudo, os autores descrevem desafios de entrega de ECMO para pacientes, visto que a demanda excedeu os recursos disponíveis. Além disso, eles enfatizam sobre outras opções de tratamento baseado em evidencias que podem também ser fornecidas para pacientes graves, dado a indisponibilidade da ECMO em muitos locais (MICHAEL M, 2020).

Um artigo aborda a eficácia do medicamento Tocilizumab no tratamento de pacientes com COVID-19 grave. O estudo foi feito com um grupo controle e um grupo com pacientes utilizando Tocilizumab, sendo avaliado a mortalidade, risco de necessidade à unidade intensiva intensa (UTI) e uso de ventilação mecânica (VM). Contudo, o estudo não teve diferenças estatísticas significantes, além do risco de admissão à UTI e uso de VM terem sido estatisticamente semelhante entre os dois grupos. Porém, as evidencias foram realizadas com baixa qualidade, não havendo, portanto, conclusões de que Tocilizumab proporcione melhora no tratamento de pacientes graves (RUAN LS, 2020).

$\mathrm{O}$ artigo relata que em infecções graves por SARS-CoV-2, ocorre uma síndrome de citocinas próinflamatórias, principalmente a interleucina 6 (IL-6), que está envolvida em um mau prognostico. Contudo, a Tocilizumab, um anticorpo monoclonal humanizado, pode ter como alvo um importante agente anti-IL-6, proposto como promissor no tratamento de COVID-19 grave. O uso de Tocilizumab, em 100 pacientes com pneumonia COVID-19 grave complicada por SDRA e síndrome hiperflamatória, mostrou uma resposta rápida e sustentada e também associada a melhora clínica. Porém, não foram relatados resultados consistentes entre os estudos comparando o uso de Tocilizumab e outros tratamentos de COVID-19 (RUAN LS, 2020).

O presente artigo explana revisões rápidas de evidencias sobre agentes potencialmente terapêuticos contra SARS-CoV-2, aos quais inclui cloroquina, hidroxicloroquina, lopinavir, ritonavir, tocilizumab, oseltamivir, interferon, atazanavir e plasma anti-SARS-CoV-2, com o intuito de guiar tomadas de decisões de profissionais de saúde, pesquisadores e comunidade acadêmica.

Ao analisar o uso da cloroquina e hidroxicloroquina, um estudo revelou que $70 \%$ dos pacientes tratados com hidroxicloroquina alcançou depuração viral em comparação para $12,5 \%$ com o grupo controle e os cinco 
pacientes tratados com hidroxicloroquina e azitromicina alcançaram depuração viral em comparação com $57,1 \%$ em pacientes tratados apenas com a hidroxicloroquina e $12,5 \%$ com o grupo de controle. Contudo, esse estudo fornece evidencias de baixa qualidade e limitações metodológicas, não apresentando veracidade para o uso desses medicamentos, portanto, nenhuma organização internacional estabeleceu recomendações específicas para gestão de ensaios clínicos de pacientes com COVID-19 com uso de cloroquina ou hidroxicloroquina (GUIDO BQ, 2020).

Além disso, drogas comumente usadas para manutenção da imunossupressão, Tacrolimus e Ciclosporina, após o transplante de órgãos sólidos, reduzem a produção de interleucina-2, um regulador de proliferação, sobrevivência e maturação para todas as células T (ANTONIO R, 2020; AKRAN A, 2020; MPHARM ZY, 2020).

Além disso, o FK506 e o ácido micofenólico inibem a produção de interleucina-17 (IL-17) com um efeito inibitório mais forte sobre Th17. Se a manifestação clínica e lesão pulmonar de COVID-19 estiverem em parte mediada pela superativação da resposta imune de células $T$, as literaturas sugerem que as condições clínicas associadas com prejuízo na resposta de células $T$, como imunossupressão em sólidos pacientes transplantados de órgãos, podem alterar o curso clínico e reduzir a taxa e a gravidade da lesão pulmonar. Embora transplantado os pacientes podem ser mais suscetíveis à infecção por SARS-CoV-2 com manifestações atípicas, o uso crônico de imunossupressores drogas podem representar um "fator de proteção" para o quadro clínico sério complicação da doença. Esta hipótese também poderia explicar por que os pacientes com linfopenia são aqueles com os piores resultados. A linfopenia pode ser causada pelo sequestro pulmonar de células $T$ hiperativadas e a imunodepressão relacionada ao medicamento pode limitar isso efeito. Além disso, os medicamentos imunossupressores podem ser uma válida Escolha "terapêutica", reduzindo a atividade do sistema imunológico de células T e evitando lesão de órgão (ANTONIO R, 2020; AKRAN A, 2020; MPHARM ZY, 2020; IPEA, 2020).

\section{CONSIDERAÇÕES FINAIS}

Dessa forma, foi concluído que existem diversos tipos de pesquisas que envolvem encontrar uma terapia medicamentosa eficiente contra o vírus COVID-19, contudo não foi possível encontrar uma pesquisa conclusiva. Sendo assim, são necessárias mais pesquisas na área a fim de desenvolver o produto que consiga tratar o vírus e pesquisas profundas que consigam associar taxas de remissão ou taxas de cura com medicações específicas.

\section{REFERÊNCIAS}

1. AKRAN A, et al. The possible of immunotherapy for COVID-19: A systematic review. International Immunopharmacology, 2020; 83: e106455.

2. ANTONIO R, et al. Immunosuppression drug-related and clinical manifestation of Coronavirus disease 2019: A therapeutical hypothesis. American Journal of Transplantation, 2020; 20(7):1947-1948.

3. BALAMURUG S, et al. Perspectives on monoclonal antibody therapy as potential therapeutic intervention for Coronavirus disease-19 (COVID-19). Medicine, 2020;38(1):10-18.

4. BENDEZU-QUISPE G, et al. Agentes potencialmente terapêuticos contra el SARS-CoV-2: Revisión de la evidencia. Rev Peru Med Exp Salud Publica, 2020;37(2):320-6

5. BRASIL. Ministério da Saúde. Sobre a doença Coronavírus. Disponível em: https://coronavirus.saude.gov.br/sobreadoenca\#: :text=O\%20que\%20\%C3\%A9\%20COVID\%2D19,Os\%20coronav\%C3\%ADrus\%20s\%C3\%A3o\&text=A\%20COVID\%2D19\%20\%C3\%A9\%20uma,infec\%C3\%A7\%C3 \%B5es\%20assintom\%C3\%A1ticas\%20a\%20quadros\%20graves. Acessado em: 15 de fevereiro de 2021.

6. BRASIL. Ministério da Saúde. Panorama dos leitos de UTI para COVID-19. Disponível em: https://coronavirus.es.gov.br/. Acessado em: 15 de fevereiro de 2021.

7. DANTE J, et al. Drug treatments comparision of plascm convalescent for covid-19: Hematol., Transfus. Cell Ther, 2020, 42(2): 113-115.

8. DAN L, et al. Efficacy of integrative Traditional Chinese and Western medicine for the treatment of patients infected with 2019 novel coronavirus (COVID-19). Medicine, 2020; 99:29

9. FRAGCOU $\mathrm{P}$, et al. Review of trials currently testing treatment and prevention of COVID-19. Clinical and Microbiological and Infection, 2020, 26(8):e19. 
10. GUIDO BQ, et al. Agentes potencialmente terapéuticos contra el SARS-CoV-2: revisión rápida de la evidencia. Revista Peruana de Medicina Experimental y Salud Publica, 2020;37(2):320-6.

11. IPEA. Centro de Pesquisa em Ciência, Tecnologia e Saúde. Quais são as pesquisas em andamento para prevenção e tratamento da Covid-19? Disponível em: https://www.ipea.gov.r/cts/pt/central-de-conteudo/artigos/artigos/198quais-sao-as-pesquisas-em-andamento-para-prevencao-e-tratamento-da-covid-20. Acessado em: 15 de fevereiro de 2021.

12. MANISHA P. Drug targets for corona vírus. Indian J Pharmacol, 2020; 52(1): 56-65

13. MATHHAYMAJ, et al. Treatment for severe acute respiratory distress syndrome from COVID-19. Lancet Respir Med, 2020, 17(3): 20-30.

14. MANISHA P, et al. Drug targets for corona virus: A systematic review. Indian J Pharmacol, 2020; 52(1): 56-65

15. MICHAEL M, et al. Treatment for severe acute respiratory distress syndrome from COVID-19. The Lancet, 2020, 8(5): 433-434.

16. MPHARM ZY, et al. Efficacy and safety of corticosteroids in COVID-19 based on evidence for COVID-19, other coronavirus infections, influenza, community-acquired pneumonia and acute respiratory distress syndrome: a systematic review and meta-analysis. Canadian Medical Association Journal (CMAJ), 2020; 192(27): e756

17. MUSA A, et al. Remdesivir for the Treatment of COVID-19: A Systematic Review of the Literature. Western Journal of Emergency Medicine, 2020; 20(4).

18. NATHAN F, et al. Systematic review of the efficacy and safety of antiretroviral drugs against SARS, MERS or COVID19: initial assessment. J Int AIDS Soc., 2020;23(4): e25489

19. NIVEDITHNA DA, et al., 2020. Efficacy and safety of convalescent plasma for severe COVID-19 based on evidence in other severe respiratory viral infections: a systematic review and meta-analysis. CMAJ 2020;192:E745-55

20. ORGANIZAÇÃO PAN-AMERICANA DE SAÚDE (OPAS). Como o vírus responsável pela COVID-19 se espalha. Disponível em: https://www.paho.org/pt/covid19\#contagio Acessado em 15 de fevereiro de 2021.

21. PHULEN S, et al. Virological and clinical cure in COVID-19 patients treated with hydroxychloroquine: A systematic review and meta-analysis. Journal of Medical Viroloy, 2020; 92(7): 776-78.

22. REED S, et al. Drug treatments for covid-19: living systematic review and network meta-analysis. British Medical Journal (BMJ), 2020;370:m2980.

23. ROMANELLI A, et al. Immunosuppression drug-related and clinical manifestation of Coronavirus disease 2019: A therapeutical hypothesis. Am J Transplant, 2020;00:1-2

24. RUAN LS, et al. Tocilizumab for severe COVID-19: a systematic review and meta-analysis. International Journal of Antimicrobial Agents, 2020, 56(3): e106103

25. SARMA $P$, et al. Virological and clinical cure in COVID-19 patients treated with hydroxychloroquine: A systematic review and meta-analysis. Journal of Medical Virology, 2020; 92(7): 776-785.

26. SI HST, et al. Medications in COVID-19 patients: summarizing the current literature from an orthopaedic perspective. Ortopedia Internacional (SICOT), 2020; 44:1599-1603.

27. SIEMIENIUK RJ, et al. Drug treatments comparision of plascm convalescent for covid-19. British Medical Journal (BMJ), 2020; 20: e2980

28. SHANMUGARAJ B, et al. Perspectives on monoclonal antibody therapy as potential therapeutic intervention for Coronavirus disease-19 (COVID-19). Asian Pac J Allergy Immunol, 2020;38(1):10-18

29. SHARON TSH, et al. Medications in COVID-19 patients: summarizing the current literature from an orthopaedic perspective. Int Orthop, 2020;44(8):1599-1603.

30. SHAO-HUAN L, et al. Tocilizumab for severe COVID-19: a systematic review and meta-analysis. Medicine, 2020; 56(3): e106103.

31. SUN YM, et al., 2020. Drug treatments for covid-19: living systematic review and network meta-analysis. International Journal of Infectious Diseases, 2020; 98:34-346.

32. SOCIEDADE BRASILEIRA DE PNEUMOLOGIA E TISIOLOGIA. Orientações da OMS para prevenção da COVID-19. Disponível em: https://sbpt.org.br/portal/covid-19-oms/. Acessado em 15 de fevereiro de 2021.

33. XUEMEI WI, et al. A systematic review and meta-analysis of the efficacy and safety of arbidol in the treatment of coronavirus disease 2019. Medicine, 2020, 99(33): e21984

34. ZHIKANG Y, et al. Efficacy and safety of corticosteroids in COVID-19 based on evidence for COVID-19, other coronavirus infections, influenza, community-acquired pneumonia and acute respiratory distress syndrome: a systematic review and meta-analysis. CMAJ, 2020; 192(27): e757 